\title{
COMUNICACIÓN
}

\section{Primer hallazgo de Spirometra mansoni en Felis domesticus de Costa Rica}

\author{
IDALIA VALERIO*, BEATRÍZ RODRÍGUEZ** y MISAEL CHINCHILLA*
}

\section{FIRST REPORT OF Spirometra mansoni IN Felis domesticus FROM COSTA RICA}

A tapeworm found in the small intestine of cats from two different zones of Costa Rica (LiberiaGuanacaste and Atenas-Alajuela), is described. According to a morphologic comparative analysis, the parasite is classified as Spirometra genus, since the uterus is coiled and the eggs have pointed ends, while in Diphyllobothrium the uterus has a rose shape and the eggs present rounded ends. The presence in the anterior part of the uterus of a variable number of coils and the undulating vagina, let us to assign the name Spirometra mansoni to this organism, which represents the first report of this parasite in Costa Rica. The importance of this parasites in the human point of view is discussed.

Key words: Cestoda, Spirometra mansoni, diphillobothrideos, cat, epidemiology, Costa Rica.

\section{INTRODUCCIÓN}

Ubicado en el grupo de los céstodos pseudophillideos y relacionado con Diphyllobothrium, el género Spirometra incluye varias especies de parásitos con ciclos de vida complejos ${ }^{1-}$ ${ }^{4}$. El término Spirometra fue sugerido en 1929 por Faust, Campbel y Kellog como Subgénero, para incluir diphillobothrideos con el útero espiralado $y$ amontonado y huevecillos con extremos aguzados, posteriormente Mueller lo eleva a género. Existen varias especies asociadas a este género presentes en el hemisferio oriental tales como Spirometra erinacei, Spirometra mansoni, Spirometra decipiens y Spirometra ranarum; por otra parte, Spirometra mansonoides y S. mansoni se encuentran en el hemisferio occidental ${ }^{4}$.

Los adultos se desarrollan en el intestino delgado de mamíferos carnívoros, especialmente cánidos y félidos. Los huevos salen con las heces al medio ambiente y en presencia de agua dulce maduran y liberan el coracidio o larva móvil. Éste penetra el primer hospedero intermediario, que es usualmente un especimen del género Cyclops y ahí se desarrolla en larva procercoide. El segundo hospedero intermediario es generalmente un anfibio y se infecta al ingerir organismos con larvas procercoides en su interior. Dentro de él se desarrolla la larva plerocercoide la cual es el estado evolutivo infectante para el hospedero definitivo ${ }^{1}$. Otros reservorios de la larva plerocercoide son reptiles, aves y mamíferos como osos, cerdos y monos ${ }^{1,2,4}$.

Morfológicamente Spirometra semeja Diphyllobothrium latum, pero tiene un tamaño mucho menor y su útero, más simple y espiralado, no alcanza la bolsa del cirro. La vagina y el cirro

\footnotetext{
* Cátedra de Parasitología. Universidad de Ciencias Médicas “Dr. Andrés Vesalio Guzmán”. San José, Costa Rica.

** Centro de Investigación en Enfermedades Tropicales (CIET). Facultad de Microbiología. Universidad de Costa Rica.
} 
se abren en forma independiente y este último se encuentra fusionado con la vesícula seminal formando una sola unidad. El escólex de Spirometra presenta dos bothrias alargadas a manera de espátulas, una en la superficie ventral y otra en la dorsal ${ }^{1,4}$.

Aunque el ser humano no es el hospedero natural de este organismo, puede ser eventualmente parasitado por él; Manson en 1882, informa sobre la primera infección en humanos y luego de este hallazgo se han descrito más casos. Los países en donde existe con más frecuencia son China, Japón, Taiwan, Corea, Vietnam, Thailandia y otros del Sureste asiático ${ }^{5}$. Esporádicamente ha sido hallado en Australia, Estados Unidos, Sudáfrica y América del Sur ${ }^{1,3,4,6}$. Spirometra no alcanza el estado adulto en el ser humano salvo en raras excepciones y cuando esto sucede, la presencia del parásito adulto no representa un serio problema médico ${ }^{4}$. Lo más frecuente es el hallazgo de larvas en diferentes tejidos, condición que se conoce como esparganosis y cuyas manifestaciones clínicas varían dependiendo de la localización del parásito ${ }^{2,4,7,8}$.

Los mecanismos de infección al ser humano incluyen la ingestión de agua contaminada con copépodos (Cyclops) infectados con la larva procercoide; el consumo de carne cruda o poco cocida de cualquiera de los reservorios de la larva plerocercoide (anfibios, reptiles, mamíferos y aves) $)^{2,4,7,8}$ y por aplicación directa sobre la piel, conjuntivas o vagina de carne cruda de sapos o serpientes a manera de cataplasmas ${ }^{4}$.

La larva se localiza con frecuencia en tejido subcutáneo, pero es posible encontrarla en otros sitios como la fascia y pared abdominal, pared toráxica, miembros inferiores y escroto. También se ha reportado su presencia en ojo, cavidad corporal, mamas, tracto urinario, sistema nervioso central, canal espinal, submucosa y pared intestinal. Las manifestaciones clínicas subcutáneas incluyen la presencia de masas palpables que pueden migrar o estar fijas, ser indoloras, enrojecidas y presentar o no prurito. La presentación subcutánea puede verse complicada por la formación de abscesos y/o hemorragia. En ojo es posible asociarlo con conjuntivitis, exoftalmia, dolor, restricción del movimiento ocular e interferencia con el campo ocular. Si la persona presenta la larva en cavidad abdominal puede palparse una masa, ocasionar peritonitis o un cuadro de abdomen agudo debido a hemorragia. La presencia de la larva en el tracto urinario puede ser causa de cistitis eosinofílica. La esparganosis escrotal se manifiesta como una masa escrotal, hematuria y disuria y en mamas como una masa palpable, a veces diagnosticada erróneamente como mastitis o cáncer. En cordón espinal se ha observado paraplejía, pérdida de la sensación debajo del nivel involucrado e incontinencia urinaria. La manifestación más severa se presenta en la esparganosis cerebral, la cual se acompaña de dolor de cabeza, hemiparesis, hemianopsia y retardo mental ${ }^{1,3-5,8,9}$.

El diagnóstico puede hacerse por biopsia o cirugía del tejido involucrado, lo que permite el diagnóstico definitivo al encontrar e identificar al parásito; a diferencia de esto, los estudios radiológicos son poco concluyentes, dada la dificultad de diferenciar al espargano de otros agentes etiológicos. En la esparganosis cerebral cobra especial importancia la tomografía axial computarizada, ya que permite ubicar la lesión y establecer posibles migraciones del parásito al observar cambios en la localización o forma de las mismas ${ }^{9}$. La serología es una ayuda importante como prueba de tamizaje y en estudios epidemiológicos, a pesar de que puede dar reacción cruzada con otras parasitosis como cisticercosis y paragonimiosis. La historia epidemiológica del paciente, la referencia de consumo de carne cruda o pobremente cocida de serpientes o ranas y la ingestión de agua no potable son factores importantes para considerar una infección por este parásito ${ }^{10}$. Los diagnósticos diferenciales incluyen la cisticercosis, el quiste hidatídico y enfermedades del sistema nervioso como la epilepsia ${ }^{1,3,4,8,9}$.

El tratamiento se basa en la remoción quirúrgica del parásito y luego de la extracción de éste es importante el seguimiento serológico del paciente. Si no se observa decrecimiento en los niveles de anticuerpos en los siguientes seis meses, debe pensarse en la posibilidad de que exista un parásito en otra localización ${ }^{4,9}$. Se ha informado que el praziquantel mata a la larva in vitro pero no es efectivo in vivo; sin embargo, Beveridge y colaboradores mencionan el tratamiento exitoso de un perro, que tenía una esparganosis proliferativa, con prazicuantel y mebendazol ${ }^{7}$.

La prevención de esta parasitosis incluye cocinar adecuadamente los alimentos, especialmente la carne de anfibios y reptiles, no beber 
agua sin hervir de ríos o lagos y la educación sanitaria pertinente $e^{2,4,9}$.

Dado lo anterior consideramos importante dar a conocer el hallazgo de dos especímenes adultos del género Spirometra sp. en el intestino delgado de dos gatos, uno procedente de Liberia (Guanacaste) y el otro de Atenas (Alajuela).

\section{MATERIAL Y MÉTODO}

Se recuperó un ejemplar de un gato proveniente de Liberia, Guanacaste y un segundo parásito proveniente de una gata de Atenas, Alajuela. Ambos gatos murieron de manera accidental y sus restos fueron sometidos a necropsia.

Los ejemplares recuperados del intestino de los animales estudiados se colocaron en solución salina, se pasaron luego a agua destilada para lograr su relajación y posteriormente fueron fijados en solución de Bouin (Sigma Diagnostics $\left.{ }^{\circledR}\right)$ de acuerdo al modo usual. El exceso de fijador se eliminó mediante lavados sucesivos en alcohol de $70^{\circ}$ y se procedió a teñirlos con carmín clorhídrico por 30 minutos. Posteriormente se lavaron en agua destilada, se decoloraron con alcohol ácido y se deshidrataron y diafanizaron en pasos sucesivos con alcoholes de graduación ascendente y mezclas de alcohol absoluto y salicilato de metilo (3:1, 2:2 y 1:3), salicilato de metilo puro y finalmente xilol. El montaje se hizo en Permount ${ }^{\circledR}$, todo de acuerdo con la recopilación de métodos de Castro y Guerrero $^{11}$. Las medidas de los proglótides se realizaron en los segmentos tratados y montados de la manera indicada, en un microscopio Olympus $\mathrm{CH} 3$ equipado con un micrómetro ocular previamente calibrado.

Varios segmentos del parásito hallado en Atenas fueron fijados con Bouin y posteriormente sometidos a las técnicas histológicas usuales para su tinción con hematoxilina-eosina.

Una porción de las heces con huevos se fijó con formalina al 10\%; otros huevos se les hizo embrionar al disponerlos en una pecera con agua de lluvia y a temperatura ambiente $\left(20-25^{\circ} \mathrm{C}\right)$. Los coracidios recuperados se utilizaron en la infección de copépodos criados en el laboratorio.

\section{RESULTADOS}

Uno de los adultos es de pequeño a mediano tamaño y mide alrededor de $30 \mathrm{~cm}$ de longitud; débilmente muscular, presenta un escólex pequeño y comprimido semejante a una espátula. El cuello es prominente, largo y delgado y los proglótides son ligeramente craspedotes. El otro ejemplar mide $35 \mathrm{~cm}$ de longitud y su escólex aplanado tiene $1,1 \mathrm{~mm}$ de longitud, 0,38 $\mathrm{mm}$ de ancho mayor y $0,1 \mathrm{~mm}$ de ancho menor, las bothrias miden $1 \mathrm{~mm}$ de largo y se encuentran una dorsal y otra ventral (Figura 1).

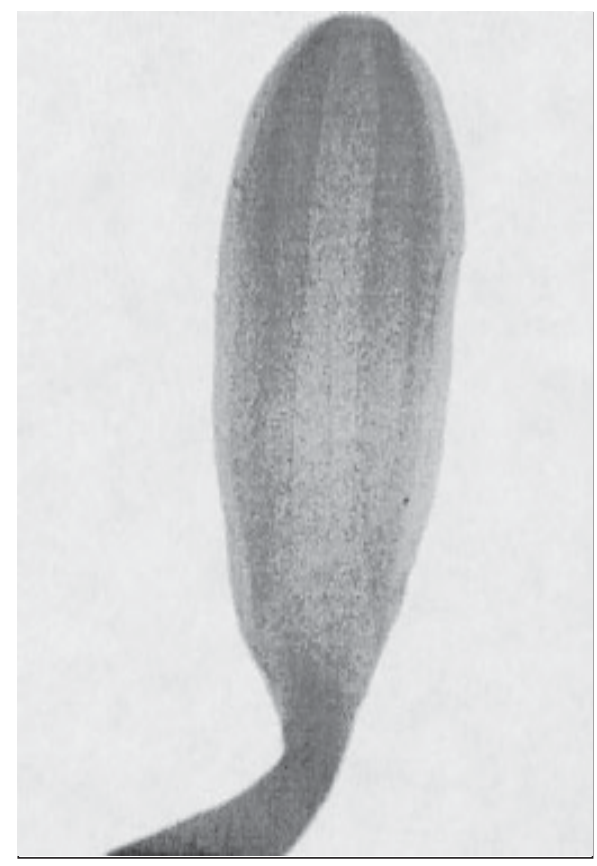

Figura 1. Escólex de Spirometra mansoni. Nótese las dos bothrias. $(0,68 \mathrm{~cm}=1 \mathrm{~mm})$.

Los proglótides maduros del ejemplar de Atenas miden 1,65 - 1,80 mm X 2,62 - 3,0 mm $(\mathrm{n}=20)$, mientras que los grávidos 2,70 - 3,75 X 2,8-4,25 mm $(\mathrm{n}=20)$. El poro genital se localiza en el tercio medio de la superficie ventral del proglótide grávido y se abre separadamente de la vagina y el cirro, este último rodeado por la vesícula seminal. El útero en forma de espiral se encuentra lleno de huevos y muestra claramente dos porciones: una anterior con una constricción al medio y otra posterior, la primera de las cuales muestra un número variable de asas (Figura 2). El cirro se abre al exterior separadamente de la vagina pero cercano al poro genital. La vagina presenta ondulaciones laterales de diferente amplitud, las cuales no se pudieron cuantificar. 


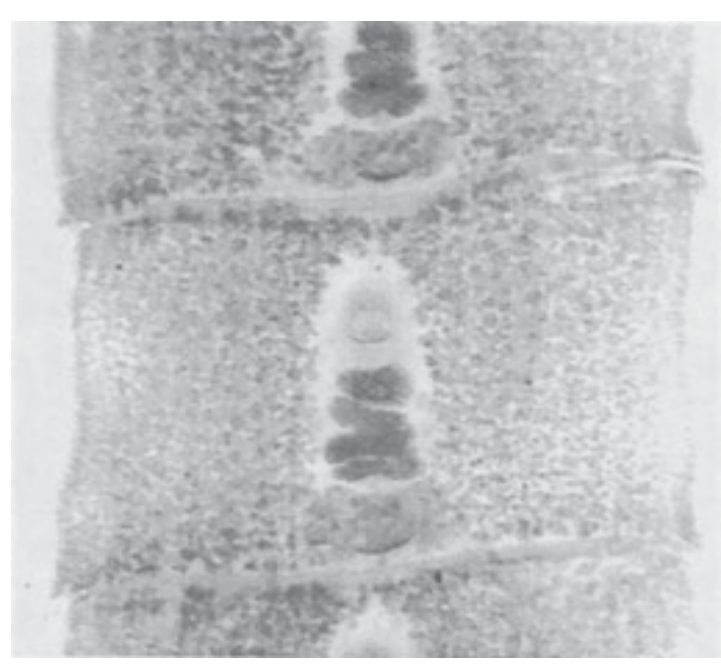

Figura 2. Proglótide grávido de Spirometra mansoni donde se observa el útero espiralado. $(1 \mathrm{~cm}=0,872 \mathrm{~mm})$.

Los huevos (Figura 3), de color pardo claro, con sus extremos aguzados y el opérculo bastante evidente, miden 57,5 - 72,5 por 30,0 - 37,5 micrómetros $(\mathrm{n}=100)$. Experimentalmente se logró su eclosión luego de 2 semanas de incubación. Los coracidios obtenidos fueron infectantes para copépodos (Cyclops sp.) criados en el laboratorio. Las larvas procercoides observadas mostraron movimientos activos.

\section{DISCUSIÓN}

En 1926, se hizo el hallazgo de un céstodo en Japón que recibió el nombre de Diphyllobothrium mansoni el cual fue luego reclasificado en el género Spirometra. Otro ejemplar de esta misma especie fue recolectado por el Dr. A. C. Foster en Panamá. En 1935, Mueller describió el hallazgo de céstodos parásitos en el intestino de varios gatos de la región de Siracusa, New York, a los que llamó Diphyllobothrium mansonoides. Posteriormente y dadas sus características diferenciales con este género, dichos organismos se ubicaron en el género Spirometra, denominándose $S$. mansonoides ${ }^{13}$. Las diferencias básicas entre ambas especies son la vagina que se localiza en posición medial para $S$. mansonoides y desciende en línea directa, a diferencia de la vagina de $S$. mansoni, que se desplaza hacia un lado de la línea media del proglótide y desciende formando ondulaciones de diferente amplitud. La

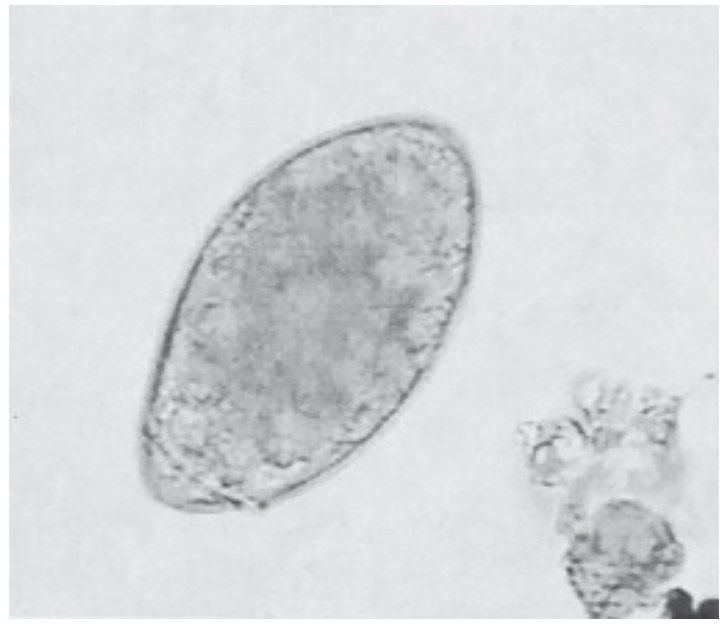

Figura 3. Huevecillo de Spirometra mansoni donde se observan los extremos puntiagudos y la presencia del opérculo. (1 cm = 10,8 micras).

otra diferencia entre ambas especies es la conformación del útero, el cual es simple, uniforme y presenta siempre dos vueltas en su parte anterior en el caso de $S$. mansonoides. En contraposición el útero de $S$. mansoni carece de uniformidad en el número de vueltas, las cuales pueden variar entre 3 y 7 asas, además de que presentan irregular disposición y tamaño ${ }^{13-16}$.

Los ejemplares hallados en Costa Rica corresponden por su morfología a parásitos del género Spirometra ya que presentan el útero en forma de espiral y huevos con los extremos puntiagudos, a diferencia del útero en roseta y huevos con extremos redondeados que se encuentra en el género Diphyllobothrium ${ }^{12}$. Además, presentan un número variable de vueltas en la parte anterior del útero, donde se cuentan entre tres y cinco asas de forma irregular y diferente longitud. La vagina, difícil de observar en los proglótides tanto maduros como grávidos, muestra ondulaciones cuando es visible. Ambas características permiten clasificar los parásitos hallados en Costa Rica como S. mansoni, siendo éste el primer reporte de este parásito en el país.

La importancia del hallazgo radica en que además de ampliar la lista de céstodos presentes en el país, representa una llamada de atención por la posibilidad de encontrar infecciones en seres humanos. Se hace necesario informar a la comunidad y a los trabajadores del área de la salud, para que al conocer el riesgo de infección, 
se mantengan alerta ante la posibilidad de la existencia de casos de esparganosis humana.

\section{RESUMEN}

Se describe un céstodo encontrado en el intestino delgado de gatos provenientes de dos zonas diferentes de Costa Rica (LiberiaGuanacaste y Atenas-Alajuela). De acuerdo con un análisis comparativo morfológico se concluye que los ejemplares hallados corresponden a parásitos del género Spirometra ya que presentan el útero espiralado y huevecillos con los extremos puntiagudos, a diferencia del útero en roseta y huevecillos con extremos redondeados que se encuentran en el género Diphyllobothrium. Dado que presentan un número variable de vueltas en la parte anterior del útero y la vagina es ondulada se clasifican como Spirometra mansoni, siendo éste el primer reporte de este parásito en el país. Se hace una corta revisión de la importancia de este parasito para el ser humano.

\section{REFERENCIAS}

1.- MIYAZAKI I. Helminthic Zoonoses. International Medical Foundation of Japan. Tokyo. 1991. 494 pp.

2.- BOTERO D, RESTREPO M. Parasitosis Humanas. 3. ed. Corporación para Investigaciones Biológicas. Medellín, Colombia. 1998. 457 pp.

3.- ATÍAS A. Parasitología Clínica Atías-Neghme. 3. ed. Editorial Publicaciones Técnicas Mediterráneo, Santiago de Chile. 1996. 618 pp.

4.- BEAVER P, JUNG R, CUPP E. Parasitología Clínica de Craig Faust. 3. ed. Editorial Masson Doyma Mexico. 2003. 823 pp.
5.- SIM S, YOU J, LEE I et al. A case of breast sparganosis. Korean J Parasitol 2002; 40: 187-9.

6.- MUELLER J F, COULSTON F. Experimental human infection with the spaganum larva of Spirometra mansonoides (Mueller, 1935) Am J Trop Med 1941; 21: 399-425.

7.- BEVERIDGE I, FRIEND S C, JEGANATHAN N, CHARLES J. Proliferative sparganosis in Australian dogs. Aus Vet J 1998; 76: 757-9.

8.- ORIHEL T, ASH L. Parasites in Human Tissues. American Society of Clinical Pathologist. Chicago, IL. Hong Kong. 1995. 386 pp.

9.- SUNDARAM C, PRASAD VS, REDDY J J M. Cerebral Sparganosis. J Ass Phys India (JAPI) 2003; 51: 1107 9.

10.- LEE K J, BAE Y T, KIM D H et al. A seroepidemiologic survey for human sparganosis in Gangweon-do. Korean J Parasitol; 40: 177-80.

11.- CASTRO A, GUERRERO-BERMÚDEZ O. Técnicas de Diagnóstico Parasitológico 1. Edición. Editorial de la Universidad de Costa Rica, San José. 2004. 99 pp.

12.- MUELLER J F. A Diphyllobothrium from cats and dogs in the Syracuse region. J Parasitol 1935; 21:11421.

13. MUELLER JF. Comparative studies on certain species of Diphyllobothrium. J Parasitol 1936; 22: 471-8.

14.- WARDLE R, MCLEOD A. The Zoology of Tapeworms. The University of Minnesota Press. Minneapolis. 1952. $780 \mathrm{pp}$.

15.- WARDLE R, MCLEOD A, RADINOVSKY S. Advances in the Zoology of Tapeworms. The University of Minnesota Press. Minneapolis. 1974. $274 \mathrm{pp}$.

16.- LOPEZ-NEYRA C R, DIAZ-UNGRIA C. Céstodos de Venezuela III. Sobre unos céstodos intestinales de reptiles y mamíferos venezolanos. Mem Soc Ciencias Nat La Salle 1957; 17: 28-63.

Agradecimiento: Este trabajo fue realizado gracias al soporte brindado por el Departamento de Investigación de la Universidad de Ciencias Médicas. 